\title{
Mechanical stent failure as a cause of life-threatening left main restenosis
}

\author{
Piotr Kübler ${ }^{1}$, Brunon Tomasiewicz ${ }^{1}$, Madeleine Johansson², Andrzej Szczepański ${ }^{3}$, Krzysztof Reczuch $^{1}$ \\ ${ }^{1}$ Department of Heart Diseases, Wroclaw Medical University, Military Hospital, Wroclaw, Poland \\ ${ }^{2}$ Students' Scientific Group of Interventional Cardiology, Department of Heart Diseases, Wroclaw Medical University, Wroclaw, Poland \\ ${ }^{3}$ Cardiology Department, Military Hospital, Wroclaw, Poland
}

Adv Interv Cardiol 2016; 12, 3 (45): 271-273

DOI: 10.5114 /aic.2016.61652

A 67-year-old woman with a history of diabetes, hypertension, severe obesity and hypercholesterolemia was admitted to our center with the diagnosis of non ST-segment elevation myocardial infarction. Electrocardiography showed $3 \mathrm{~mm}$ ST-segment depressions in leads I, aVF, and V3-V6 and elevation in lead aVR. Troponins were positive and ejection fraction assessed in echocardiography was $50 \%$, without valve abnormalities. Five months earlier, the patient underwent elective percutaneous coronary intervention $(\mathrm{PCl})$ of the left main coronary artery (LMCA) and left anterior descending artery (LAD) using 2 overlapping everolimus-eluting stents (Promus-Premier, Boston Scientific): $3.5 / 32 \mathrm{~mm}$ to the LMCA/LAD and $2.5 / 32 \mathrm{~mm}$ to the LAD with non-compliant $4.0 \mathrm{~mm}$ balloon high pressure postdilatation in the LMCA and with full expansion, an optimal angiographic result, unfortunately without intravascular ultrasound (IVUS) assistance. Qualification for PCl at that time followed the Heart Team meeting and discussion with the patient (low values of both Syntax-Score (21) and EuroSCORE II (0.9\%)) - she preferred the option of PCI more than coronary artery bypass grafting (CABG).

Coronary angiography revealed critical in-stent restenosis in the calcified LMCA (Figure 1) with diffuse non-significant changes in the LAD, circumflex artery and right coronary artery. During angiography severe chest pain and hemodynamic instability occurred, so immediate in-stent balloon angioplasty with 3.0/15 mm and $3.5 / 15 \mathrm{~mm}$ balloons to $10 \mathrm{~atm}$ was performed, enough to improve the flow in the left coronary artery and with clinical stability. However, IVUS examination in the LMCA showed some features of mechanical stent failure - separation of struts on one side of the stent circumference, in place of vessel calcification, which could be the most likely cause of restenosis. Unfortunately, because of the lack of baseline IVUS, we could not clearly diagnose the type of stent deformation. We decided to implant another Biolimus-eluting stent, 3.5/14 mm with $4.0 / 8 \mathrm{~mm}$ non-compliant balloon postdilatation, and the final result was perfect (Figure 1).

Mechanical stent failure (MSF) is a rare but potentially catastrophic complication in complex $\mathrm{PCl}$ procedures. The most common types are longitudinal stent deformation, stent fracture, stent underexpansion and stent recoil. The reported general MSF rate of everolimus-eluting stents is about $9.6 \%$, the longitudinal stent deformation rate is about $5.4 \%$, and the stent fracture rate is about $2.9 \%[1,2]$. However, in many cases angiography alone does not reveal the presence of MSF. Intravascular imaging techniques (IVUS, optical coherence tomography) appear to be more sensitive methods and are more useful in the diagnosis of stent deformation. Because IVUS is not routinely performed, MSF rates are likely to be underestimated [1, 3]. Mechanical stent failure is associated with more frequent occurrence of major adverse cardiac events (in-stent restenosis, stent thrombosis, target lesion revascularization) [4]. Current data show that longitudinal stent deformation risk factors include everolimus stent, Guideliner use, use of postdilatation balloons and multiple stent implantation [2], and stent fracture risk factors include vessel tortuosity, longer stent length, overlapping stenting, calcified and ostial lesions and high deployment pressure $[3,4]$. In our case, the stent was placed ostially, in a calcified LMCA, followed by high pressure postdilatation, which may explain the mechanical failure. Additional percutaneous intervention, stent implantation or optionally CABG is the treatment of choice in such a complication. The described case underscores

Corresponding author:

Piotr Kübler MD, Department of Heart Diseases, Wroclaw Medical University, Military Hospital, 5 Weigla St, 50-981 Wroclaw, Poland, phone: +48 7176604 52, e-mail: pkubler75@gmail.com

Received: 5.10.2015, accepted: 24.12.2015. 

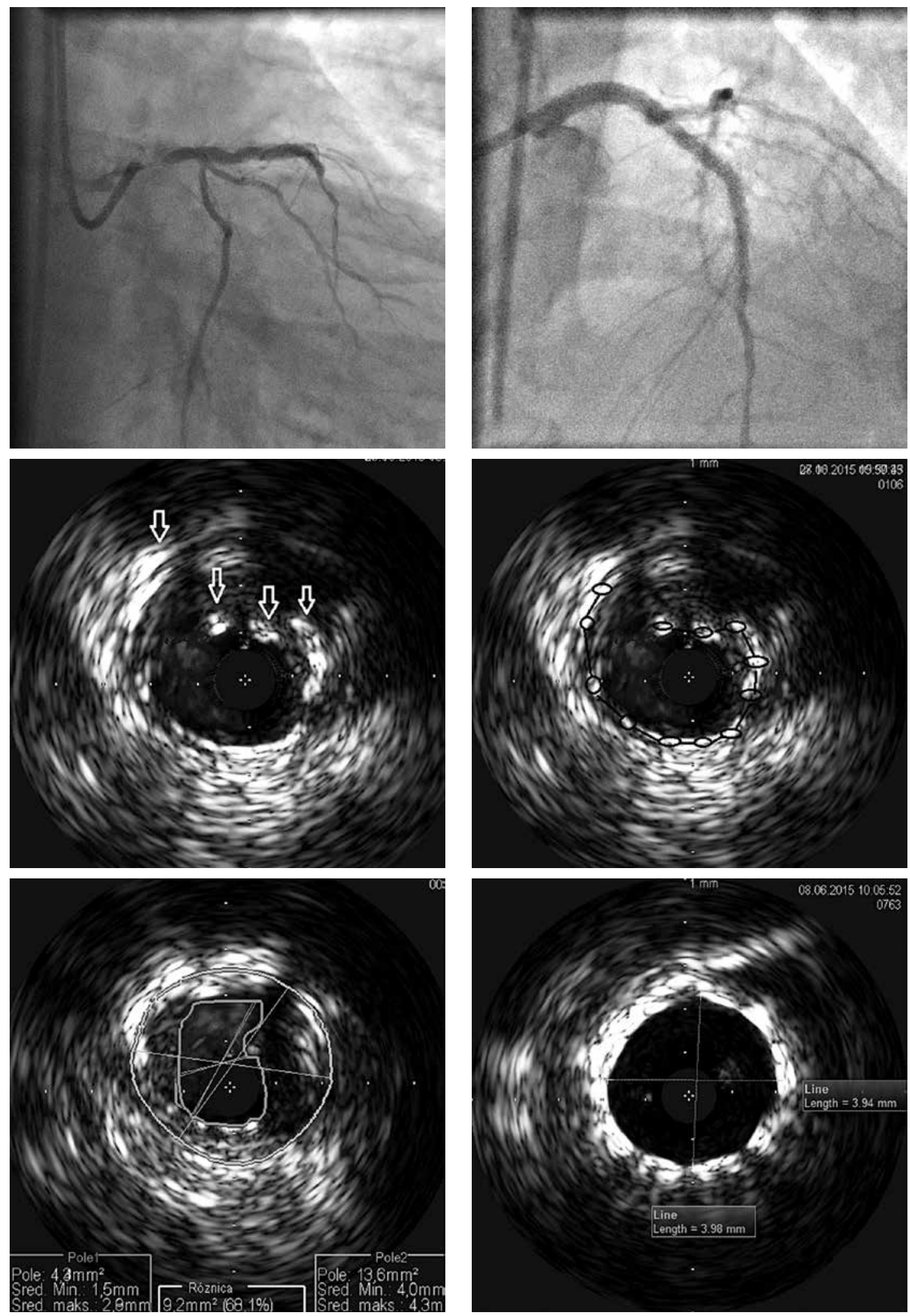

Figure 1. Coronary angiography on admission showing critical in-stent restenosis in left main coronary artery (upper left side) and final result after additional stent implantation (upper right side). Beneath intravascular ultrasound (IVUS) study with features of mechanical stent failure: separation of struts (white arrows) on one side of the stent circumference (black lines) at $4 \mathrm{~mm}$ from the left main ostium. Lower left: cross-section at $2 \mathrm{~mm}$ from the left main ostium with reference area at the level of significant restenosis (minimal lumen area $1.5 \mathrm{~mm} / 2.8 \mathrm{~mm}\left(4.3 \mathrm{~mm}^{2}\right)$; left main cross-section area $4.0 \mathrm{~mm} / 4.3 \mathrm{~mm}\left(13.6 \mathrm{~mm}^{2}\right) ; 68.1 \%$ stenosis). Final result after another stent implantation - lower right 
the importance of IVUS-guided LMCA stenting to obtain an optimal result of the procedure.

\section{Conflict of interest}

The authors declare no conflict of interest.

\section{References}

1. Inaba S, Mintz GS, Yun KH, et al. Mechanical complications of everolimus-eluting stents associated with adverse events: an intravascular ultrasound study. Eurolntervention 2014; 9: 1301-8.

2. Arnous S, Shakhshir N, Wiper A, et al. Incidence and mechanisms of longitudinal stent deformation associated with Biomatrix, Resolute, Element, and Xience stents: angiographic and case-by-case review of 1,800 PCls. Catheter Cardiovasc Interv 2015; 86: 1002-11.

3. Nakazawa G, Finn AV, Vorpahl M, et al. Incidence and predictors of drug-eluting stent fracture in human coronary artery a pathologic analysis. J Am Coll Cardiol 2009; 54: 1924-31.

4. Kuramitsu S, Iwabuchi M, Haraguchi T, et al. Incidence and clinical impact of stent fracture after everolimus-eluting stent implantation. Circ Cardiovasc Interv 2012; 5: 663-71. 